\title{
5 giorni di antibiotico per le polmoniti non complicate sono sufficienti: i risultati di non inferiorità del SAFER RCT
}

Pernica JM, Harman S, Kam AJ, et al.

Short-Course Antimicrobial Therapy for Pediatric Community-Acquired Pneumonia: The SAFER Randomized Clinical Trial JAMA Pediatr. 2021;175(5):475-482. doi: 10.1001/jamapediatrics.2020.6735

Le più importanti linee guida per il trattamento della polmonite acquisita in comunità (CAP) indicano l' amoxicillina come farmaco di prima scelta, tuttavia mancano indicazioni evidence-based circa la durata. Questo studio realizzato in 2 dipartimenti di emergenza in Canada ha randomizzato 281 bambini di età compresa tra 6 mesi e 10 anni con CAP senza necessità di ricovero, al trattamento con amoxicillina ad alte dosi per 5 giorni vs i tradizionali 10 giorni. In termini di guarigione clinica entrambi i gruppi hanno presentato risultati paragonabili. In realtà l'analisi "per protocol", raccomandata per un disegno di "non-inferiorità", non ha fornito formalmente questo risultato. I criteri di reclutamento esclusivamente clinici (eventuali indagini erano opzionali) rispecchiano bene la realtà delle cure primarie, e i risultati, pur con alcuni limiti, suggeriscono che nella CAP non complicata la terapia breve debba essere considerata nelle linee guida.

5 days of antibiotic for uncomplicated pneumonia is enough: the non-inferiority results of the SAFER RCT

The most important guidelines for the treatment of community-acquired pneumonia (CAP) indicate amoxicillin as the drug of first choice, however there is a lack of evidence-based indications about the duration of this therapy. This study conducted in 2 emergency departments in Canada randomized 281 children aged 6 months to 10 years with CAP without the need for hospitalization to treatment with high-dose amoxicillin for 5 days versus a traditional 10 day therapy. In terms of clinical recovery, both groups presented comparable results. In fact, the "per protocol" analysis, recommended for a "non-inferiority" design, did not formally provide this result. The exclusively clinical recruitment criteria (any investigations were optional), well reflect the reality of primary care, and the results, albeit with some limitations, suggest that in uncomplicated CAP, brief therapy should be considered in the guidelines.

\section{Metodo}

\section{Obiettivo (con tipo studio)}

Studio randomizzato controllato di non inferiorità multicentrico in doppio cieco per determinare se la terapia di 5 giorni con amoxicillina ad alte dosi dà risultati clinici non inferiori alla terapia di 10 giorni nelle polmoniti acquisite in comunità (CAP).

\section{Popolazione}

Bambini di età compresa tra 6 mesi e 10 anni con CAP senza necessità di ricovero, afferenti a due dipartimenti di emergenza (Ontario, Canada).

Per la diagnosi di CAP dovevano essere soddisfatti tutti i seguenti criteri:

- febbre $\left(>37.5^{\circ} \mathrm{C}\right.$ ascellare, $>37.7^{\circ} \mathrm{C}$ orale $\mathrm{o}>38^{\circ} \mathrm{C}$ rettale $)$ nelle 48 ore precedenti la presentazione;

- uno qualsiasi tra: tachipnea; tosse all'esame o in anamnesi; dispnea; reperti clinici compatibili con polmonite (crepitii focali, respirazione bronchiale, ecc.).

- radiografia del torace compatibile con CAP batterica;

- diagnosi di CAP primaria.

Criteri di esclusione: patologie che potevano predisporre a malattia severa e/o polmonite ad eziologia atipica:

- empiema o polmonite necrotizzante, malattie polmonari preesistenti, cardiopatie congenite, neoplasie maligne, storia di aspirazione, immunodeficienze, insufficienza renale, mononucleosi; - trattamento con beta lattamici nel giorno precedente all'arruolamento, oppure 5 giorni di beta lattamici 72 ore prima dell'arruolamento o terapia EV con cefalosporina o azitromicina durante l'accesso in pronto soccorso; trattamento con warfarin o tetracicline;

- ospedalizzazione prolungata nei 2 mesi precedenti, CAP nel mese precedente, ascesso polmonare nei 6 mesi precedenti; - allergia a penicillina.

\section{Intervento}

Terapia breve: amoxicillina $90 \mathrm{mg} / \mathrm{kg} / \mathrm{die}$ per 5 giorni in 3 dosi quotidiane (TID) seguito da placebo per 5 giorni TID.

\section{Outcome/Esiti}

Outcome primario: guarigione clinica definita dai seguenti parametri:

1) iniziale miglioramento nei primi 4 giorni dopo l'arruolamento (incluso sfebbramento);

2) significativo miglioramento dello sforzo respiratorio e scomparsa della tachipnea tra il giorno 14 e 21;

3) non più di una puntata febbrile come risultato di una possibile malattia batterica dal giorno 4 alla fine del follow-up clinico;

4) nessuna necessità di associazione con altra terapia antimicrobica o ospedalizzazione per persistenza o peggioramento di malattia delle basse vie aeree;

L'outcome primario è stato misurato tramite valutazione delle risposte fornite dai caregiver nel diario tra 3 e 5 giorni, tra 7 e 10 giorni di follow-up e con visite ambulatoriali tra i 14 e i 21 giorni. 
Nel corso dello studio è stato creato un nuovo outcome secondario post hoc definito come iniziale miglioramento dopo 4 giorni dall'arruolamento e nessuna necessità di altri interventi: terapia antimicrobica o ospedalizzazione.

Outcome secondari: numero di giorni di assenza da scuola dei bambini, numero di giorni di assenza dal lavoro dei genitori, numero di giorni di reazione modesta al farmaco, numero di reazioni severe al farmaco, aderenza alla terapia e ricorrenza di malattia respiratoria di presunta natura batterica dopo la visita finale ed entro un mese dall'arruolamento.

\section{Tempo}

Studio pilota condotto dal 1/12/2012 al 31/3/2014 (arruolamento di 60 pazienti). Studio principale con arruolamento dal 1/1/2017 al 31/12/2019, interrotto per mancanza di fondi.

\section{Risultati principali}

Per lo studio sono stati arruolati 281 pazienti [ $57 \%$ maschi, di 2 pazienti non erano disponibili i dati; età media 2.6 anni (6 mesi-10 anni); 140 nel gruppo di intervento - 141 nel gruppo di controllo]. All'analisi "per protocol" (PP: pazienti aderenti al trattamento) la guarigione clinica tra il giorno 14 e 21 è risultata simile nei 2 gruppi: 101 su 114 pazienti (88.6\%) nel gruppo di intervento e 99 su 109 (90.8\%) nel gruppo di controllo. All'analisi per "intention to treat" (ITT) la guarigione clinica è stata osservata in 108 pazienti su $126(85.7 \%)$ del gruppo di intervento e in $106 \mathrm{su} 126$ (84.1\%) del gruppo di controllo. All'analisi "strict per-protocol" (pazienti aderenti al trattamento con conferma radiologica di CAP) la guarigione clinica è stata documentata in 73 su 82 pazienti (89\%) del gruppo di intervento e in 74 su 83 (89.1\%) del gruppo di controllo.

\section{Conclusioni}

Questo RCT di non-inferiorità ha rilevato che, in una popolazione di bambini precedentemente sani e che non richiedono ospedalizzazione, un trattamento per CAP con amoxicillina ad alte dosi per 5 giorni determina risultati clinici paragonabili a quelli ottenuti con il trattamento standard (10 giorni di amoxicillina ad alte dosi). Gli autori ritengono che le linee guida per la gestione pratica delle CAP in età pediatrica dovrebbero raccomandare il trattamento di 5 giorni con amoxicillina, anche in conformità coi principi dell'appropriatezza d'uso degli antibiotici.

\section{Altri studi sull'argomento}

Le linee guida della Pediatric Infectious Diseases Society e dell'Infectious Diseases Society of America [1] indicano l'amoxicillina come antibiotico di prima scelta per una durata di 10 giorni; la British Thoracic Society [2] indica la possibilità di una terapia antibiotica di 5 giorni con amoxicillina come farmaco di prima scelta; l'Organizzazione Mondiale della Sanità $[3,4]$ e il National Institute for Health and Care Excellence [5] indicano l'amoxicillina come farmaco di prima scelta e una durata della terapia di 5 giorni. Una revisione sistematica Cochrane aggiornata al 2021 indica la mancanza di prove di efficacia per supportare o contestare l'utilizzo di antibiotici in bambini di 2-59 mesi di età con polmonite non grave con wheezing [6]. Una revisione Cochrane del 2013 (non più aggiornata) ha raccolto 29 studi (14.188 bambini) con l'obiettivo di identificare le terapie antibiotiche efficaci nella CAP. Nessuno studio ha confrontato un antibiotico verso placebo. Il confronto tra terapia con amoxicillina vs cotrimossazolo non ha evidenziato differenze (tre studi). Nelle CAP gravi una terapia per os (sei studi) con amoxicillina o cotrimossazolo vs terapia iniettabile con penicillina ha determinato esiti similari tra i due gruppi, mentre (uno studio) i tassi di mortalità erano più elevati nel gruppo trattato con cloramfenicolo vs penicillina/ampicillina + gentamicina [7]. Un RCT in doppio cieco controllato (amoxicillina contro placebo) su 1.126 bambini con età inferiore a 6 anni in Malawi ha confrontato la terapia con amoxicillina verso il placebo per CAP non grave diagnosticata clinicamente. Dopo 3 gg di cura erano presenti sintomi nel $7 \%$ del gruppo placebo contro il $4 \%$ del gruppo trattato con una differenza assoluta del rischio del 3\% (IC 95\% 0.4\%, 5.7\%). A 14 giorni non c'erano differenze di esito tra i due bracci; nel confronto con il gruppo placebo il numero necessario di bambini da trattare nel gruppo amoxicillina per avere una guarigione era $33[8]$.

\section{Che cosa aggiunge questo studio}

Il trial, dal disegno metodologico rigoroso, rispecchia un setting di cure primarie e indica la necessità di rivalutare le linee guida della CAP soprattutto riguardo la durata della terapia antibiotica che dovrebbe essere abbreviata; inoltre conferma l'amoxicillina come farmaco di prima scelta.

\section{Commento}

\section{Validità interna}

Disegno dello studio: la randomizzazione, stratificata per sito, è stata ottenuta in modo adeguato. Lo studio è stato condotto in doppio cieco: né pazienti, né personale di cura erano a conoscenza del braccio di trattamento. È uno studio di non inferiorità, con margine di non inferiorità fissato a priori al $7.5 \%$ e in cui l'analisi dei dati relativi agli esiti primari e secondari è stata eseguita sia per ITT che PP. Come riportato in discussione dagli stessi autori, nell'analisi PP dell'esito primario, poiché il limite di confidenza (CL) unilaterale del $97.5 \%$ circa la differenza di rischio ha attraversato il margine di non-inferiorità del 7.5\%, non può essere fatta una conclusione formale di non-inferiorità, tuttavia nell'analisi ITT, il trattamento breve è risultato statisticamente non inferiore. Tuttavia, poiché l'analisi ITT tende ad attutire le differenze, dovrebbe essere da privilegiare negli studi di superiorità, mentre per studi di equivalenza o non inferiorità sarebbe meglio non utilizzarla, oppure presentarla accanto ad altri tipi di analisi. Dei 281 pazienti arruolati, $10.3 \%$ sono stati persi al follow up, in modo bilanciato nei due gruppi, ma senza motivazioni esplicitate. Il numero di partecipanti è risultato basso rispetto ai potenzialmente elegibili, infatti dei 5.406 pazienti diagnosticati con CAP, solo 281 sono stati arruolati: 1.910 sono stati esclusi in quanto non elegibili o per mancata autorizzazione dei caregiver; ben 3.215 non sono stati arruolati senza ulteriore specificazione delle motivazioni. Il disegno dello studio prevedeva l'arruolamento di pazienti con età compresa tra 6 mesi e 10 anni, renden- 
do la popolazione in esame estremamente disomogenea sia come prevalenza di malattia infettiva batterica, che come eziologia. Sono state eseguite analisi per sottogruppo: età ( $<5$ vs $\geq 5$ anni), proteina C-reattiva salivare ( $<30 \mathrm{vs} \geq 30 \mathrm{pg} / \mathrm{mL}$ ), e riscontro di virus o mycoplasma (test negativo vs positivo) nell'aspirato nasale; i risultati sono riportati nel supplemento, tuttavia non sono emerse differenze significative anche per il basso numero di casi nei sottogruppi. Si segnala che tutti i test di laboratorio erano opzionali; presso uno dei 2 centri veniva eseguito un tampone naso-faringeo per un pannello di virus respiratori e, a posteriori un test multiplex polymerase chain reaction assay per mycoplasma pneumoniae e chlamydia pneumoniae; inoltre su un campione di saliva veniva determinato il valore di proteina $C$ reattiva (PCR). L'età prescolare dei soggetti arruolati, l'alta percentuale di isolamenti virali nel tampone naso-faringeo, i bassi valori di PCR nei campioni salivari e la non significatività di parte dei radiogrammi del torace agli occhi del radiologo fanno sospettare un'alta percentuale di CAP di origine virale nei bambini arruolati in entrambi i gruppi.

Esiti: ben definiti e di rilievo clinico, sebbene la valutazione del paziente sia stata condotta nei primi 10 giorni esclusivamente tramite contatti telefonici con i genitori e solo successivamente con visita tra il giorno 14 e il giorno 21. Da sottolineare che gli autori stessi hanno ritenuto opportuno aggiungere nell'analisi post hoc un esito secondario che riguardava la "guarigione clinica senza necessità di ulteriori interventi terapeutici" per identificare i soggetti che nella pratica clinica sarebbero stati considerati guariti (mentre per le strette definizioni di "guarigione" del trial rientravano nel fallimento terapeutico).

Conflitto di interesse: due autori dichiarano conflitto di interesse con ditte di farmaci.

\section{Trasferibilità}

Popolazione studiata: è simile a quella che viene normalmente valutata negli ambulatori pediatrici di primo livello; da sottolineare che la copertura vaccinale in Italia a 24 mesi per lo pneumococco è del 92\% (dati del 2019 https://www.epicentro.iss.it/ vaccini/dati_Ita\#pneumo), mentre la copertura in Canada nei 6 anni precedenti allo studio era del 74-78\%.

Tipo di intervento: applicabile anche in Italia, anche se i risultati dello studio non possono al momento essere considerati come raccomandazioni o protocolli diagnostico terapeutici assistenziali. Il Sistema Nazionale Linee Guida dell'Istituto Superiore della Sanità (https://snlg.iss.it/) ad oggi non ha pubblicato alcuna linea guida sulle polmoniti in età pediatrica. É auspicabile che le Società scientifiche italiane colmino al più presto questa importante lacuna alla luce delle modificazioni epidemiologiche delle CAP dopo l'introduzione del vaccino antipneumococcico.

1. Bradley JS, Byington CL, Shah SS et al.; Pediatric Infectious Diseases Society and the Infectious Diseases Society of America. The management of community-acquired pneumonia in infants and children older than 3 months of age: clinical practice guidelines by the Pediatric Infectious Diseases Society and the Infectious Diseases Society of America. Clin Infect Dis. 2011;53(7):e25-76. doi: 10.1093/cid/cir531.

2. Harris M, Clark J, Coote N et al.; British Thoracic Society Standards of Care Committee. British Thoracic Society guidelines for the management of community acquired pneumonia in children: update 2011. Thorax. 2011;66 Suppl 2:ii1-23. doi: 10.1136/thoraxjnl-2011-200598.

3. World Health Organization. Revised WHO classification and treat- ment of childhood pneumonia at health dacilities - evidence summaries. Geneva: WHO; 2015

4. Mathur S, Fuchs A, Bielicki J et al. M. Antibiotic use for community-acquired pneumonia in neonates and children: WHO evidence review. Paediatr Int Child Health. 2018;38(sup1):S66-S75. doi: 10.1080/20469047.2017.1409455.

5. Murphy S, Thomson L. NICE community-acquired pneumonia guideline review. Arch Dis Child Educ Pract Ed. 2021 Oct;106(5):296-298. doi: 10.1136/archdischild-2020-319376.

6. Lassi ZS, Padhani ZA, Das JK, et al. Antibiotic therapy versus no antibiotic therapy for children aged 2 to 59 months with WHO-defined non-severe pneumonia and wheeze. Cochrane Database Syst Rev. 2021 Jan 20;1(1):CD009576. doi: 10.1002/14651858.CD009576.7.

7. Lodha R, Kabra SK, Pandey RM. Antibiotics for community-acquired pneumonia in children. Cochrane Database Syst Rev. 2013 Jun 4;2013(6):CD004874. doi: 10.1002/14651858.CD004874.pub4.

8. Ginsburg AS, Mvalo T, Nkwopara E, et al. Placebo vs amoxicillin for nonsevere fast-breathing pneuamonia in Malawian children aged 2 to 59 months: a double-blind, randomized clinical noninferiority trial. JAMA Pediatr. 2018;173 (1):21-28. doi:10.1001/jamapediatrics.2018.3407

\section{Scheda redatta dal gruppo di lettura di Reggio Emilia:}

Francesca Bontempo, Carlo Boni, Jennifer Chiarolanza, Angelo Cigarini, Elena Corbelli, Annalisa Correggi, Anna Maria Davoli, Anna Rita Di Buono, Elena Ferrari, Monica Malventano, Elena Manzotti, Maddalena Marchesi, Gino Montagna, Luciana Monti, Rosaria Ollari, Luisa Seletti, Mariassunta Torricelli, Maria Candida Tripodi, Daniela Vignali, Marialuisa Villani, Costantino Panza. 